\title{
A GENERAL REGULARITY THEOREM FOR SEMILINEAR HYPERBOLIC WAVES IN ONE SPACE DIMENSION
}

\author{
BY JEFFREY RAUCH AND MICHAEL REED
}

We study the propagation of singularities for semilinear strictly hyperbolic systems in one space dimension

$$
D_{i} u_{i}=f_{i}(x, t, u), \quad u_{i}(x, 0)=u_{i}^{(0)}(x),
$$

$i=1, \ldots, m$, where the vector fields $D_{i}$ and the functions $f_{i}$ are $C^{\infty}$. In [1] we presented a simple example which shows that the propagation of singularities for (1), is, in general, not the same as for the special case where the $f_{i}$ are linear in $u$. In [2] we used elementary methods to study this problem when the initial data are piecewise smooth. We report here on results which permit general initial data $u^{(0)} \in$ $H_{\text {loc }}^{s}, s>1 / 2$.

The propagation of singularities for (1) is governed by two general principles:

TREE LAW. When two or more singularity bearing characteristics intersect, the point of intersection becomes a source of singularities travelling, in general, in all forward characteristic directions from the point.

SUM LAW. When $H^{s}$ singularities collide with $H^{\tau}$ singularities, the new singularities produced will have strength $H^{s+\tau}$ (this must be interpreted in a suitable microlocal sense).

Let $S^{(0)}$ denote the union of forward characteristics from the singular support of the initial data. Let $S^{(1)}$ denote the union of forward characteristics from points of intersection in $S^{(0)}$. Continue defining $S^{(l)}$ in this way and set $S=$ closure $\bigcup^{\infty} S^{(l)}$. The first general principle indicates that, in general, $S$ will be the singular support of $u$. The second general principle predicts the regularity of $u$ at various points in $S$.

ExAmPle 1. Let $m=3, D_{1}=\partial / \partial t+\partial / \partial x, D_{2}=\partial / \partial t-\partial / \partial x, D_{3}=\partial / \partial t$ and suppose that $u^{(0)}$ is $H^{s}$ on $[a, b]$ and $C^{\infty}$ outside $[a, b]$. Then the two general principles predict the regularity depicted in Figure 1 . If the $f_{i}$ are linear, then $u$ will be $C^{\infty}$ in the regions labelled $H^{2 s}, H^{3 s}, \ldots$

ExAmple 2. Suppose that $m=4$ and that there are two rightward moving and two leftward moving characteristics. Suppose that the data is $C^{\infty}$ except at two points $x_{1}$ and $x_{2}$.

Received by the editors May 4, 1981.

1980 Mathematics Subject Classification. Primary 35L67. 


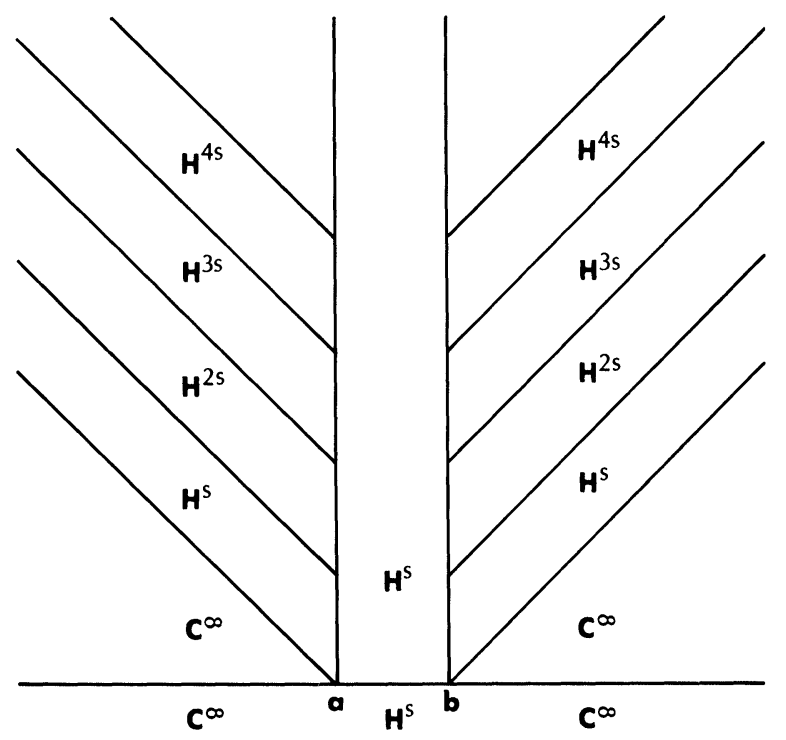

FIGURE 1. Regularity in Example 1

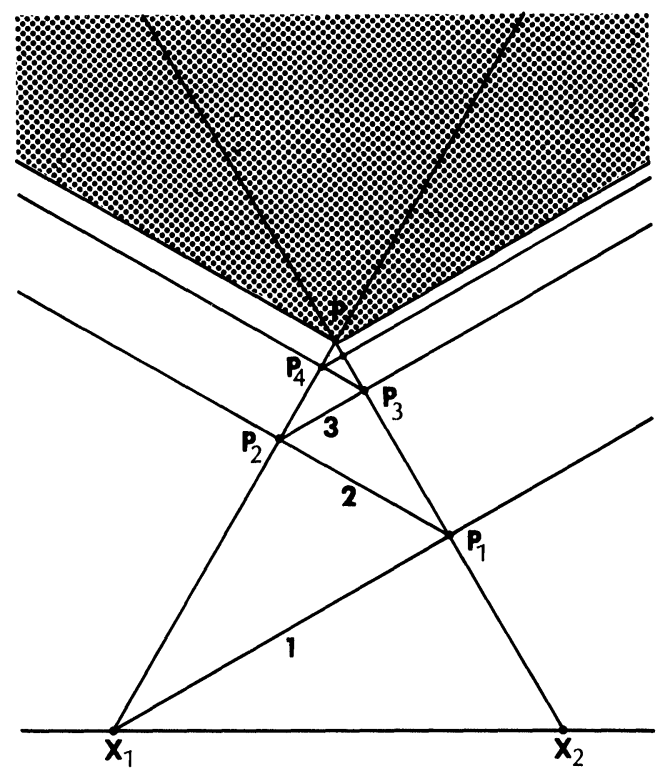

Figure 2 
The fast rightward characteristic from $x_{1}$ (labelled 1 in Figure 2) intersects the slow leftward characteristic from $x_{2}$ at $P_{1}$. A new fast leftward singularity will be produced (labelled 2) and at $P_{2}$ a new fast rightward singularity will be produced. In this way a sequence of intersection points $P_{1}, P_{2}, P_{3}, \ldots$ in $S$ will be produced such that $P_{n} \rightarrow P$. It can be shown that whenever $S$ contains such a limit point $P$, it will also contain the entire solid forward cone from $P$ (shaded). Thus initial singular support of only two points can produce a solid forward cone in the singular support of the solution if $m \geqslant 4$.

In order to prove theorems about these phenomena, we develope the harmonic analysis of a family of spaces of distributions appropriate to the problem. The usual spaces defined by decay of the Fourier transform in cones do not provide fine enough tools because one needs not only the behavior on Char $D_{i}$ and off Char $D_{i}$ but also the behavior up to Char $D_{i}$. Further, it is important that the spaces be invariant under composition with $C^{\infty}$ maps so that $f(x, t, u)$ has the same regularity as $u$.

Define

$$
\left(H^{s}\right)_{\partial / \partial x_{1}}^{k}\left(\mathbf{R}^{2}\right)=\left\{u \in S^{\prime}\left(\mathbf{R}^{2}\right) \mid\left(1+\xi^{2}\right)^{s / 2}\left(1+\xi_{1}^{2}\right)^{k / 2} u\left(\xi_{1}, \xi_{2}\right) \in L^{2}\left(\mathbf{R}^{2}\right)\right\} .
$$

If $x \in \mathbf{R}^{2}$ and $D$ is a $C^{\infty}$ vector field, then by change of variables one can define local spaces $\left(H^{s}\right)_{D}^{k}(p)$ in a natural way. If $k$ is a nonnegative integer, this is just the set of $u$ such that $u, D u, \ldots, D^{k} u$ are all in $H^{s}$ locally at $p$. In [1] we showed that $\bigcap_{k}\left(H^{s}\right)_{D}^{k}(p)$ is the right space for describing the regularity of $u$ at points $p$ where there are singularities moving in only one direction $D(p)$. To describe singularities moving in $m$ directions at $p$ we define the following spaces: Let $r_{i}>1 / 2, i=1, \ldots, m$, be given, $\rho=\min \left\{r_{i}+r_{j}, i \neq j\right\}$. Then

$$
A(\vec{r} ; p) \equiv \underset{\left\{i \mid r_{i}<\rho\right\}}{\bigoplus}\left(H^{r_{i}}\right)_{D_{i}(p)}^{\rho-r_{i}}(p)
$$

This is just the set of $u$ which can be written $u=\Sigma^{m} u_{i}$ with $u_{i} \in\left(H^{r_{i}}\right)_{D_{i}}^{\rho-r_{i}}(p)$.

THEOREM 1. $A(\vec{r} ; p)$ is an algebra invariant under $C^{\infty}$ maps.

One thinks of $A(\vec{r} ; p)$ as the regularity corresponding to the phrase "singularities of strength $r_{i}$ travelling in direction $D_{i}(p)$ at $p$." This interpretation suggests the following rule for predicting the regularity of $u$ from that of its Cauchy data.

Let $\Gamma^{i}(q, p)$ denote the $i$ th characteristic between $q$ and $p$. Let $(c, d)$ be an interval on which initial data $u^{0} \in H_{10 c}^{s}, s>1 / 2$, are given and let $R$ be the set of points whose initial interval of dependence is contained in $(c, d)$. Suppose $\tau$ : $(c, d) \rightarrow(1 / 2, \infty)$ is a function such that $p_{0} \in(c, d)$ implies $u^{0} \in H^{\tau\left(p_{0}\right)}\left(p_{0}\right)$. 
For any $p$ on the $i$ th forward characteristic from $p_{0}$ we define $\sigma_{i}^{(0)}(p)=\tau\left(p_{0}\right)$. Inductively, we define,

$$
\sigma_{i}^{(l)}(p)=\inf _{q \in \Gamma^{i}\left(p_{0}, p\right)}\left\{\sigma_{i}^{(l-1)}(q), \min _{\substack{i, j, k \\ \text { distinct }}}\left(\sigma_{j}^{(l-1)}(q)+\sigma_{k}^{(l-1)}(q)\right)\right\}
$$

and set

$$
\sigma_{i}(p) \equiv \lim _{l \rightarrow \infty} \sigma_{i}^{(l)}(p)
$$

$\vec{\sigma}(\cdot)=\left(\sigma_{1}(\cdot), \ldots, \sigma_{m}(\cdot)\right)$ is called an index of regularity for $u$.

THEOREM 2. Let $u \in L^{\infty}(R)$ be a distribution solution of (1) in $R$ with initial data $u^{0} \in H_{10}^{s}, s>1 / 2$. Let $\vec{\sigma}$ be an index of regularity for $u$. Then $u \in$ $A(\vec{\sigma}(p) ; p)$ for each $p$ in $R$.

The main ingredients in the proof are refinements of the harmonic analysis indicated in Theorem 1 and local nonlinear elliptic and hyperbolic regularity theorems adapted to the algebras $A(\vec{r} ; p)$.

\section{REFERENCES}

1. J. Rauch and M. Reed, Propagation of singularities for semilinear hyperbolic equa. tions in one space variable, Ann. of Math. (2) 111 (1980), 531-552.

2. Jump discontinuities of semilinear, strictly hyperbolic systems in two variables, Comm. Math. Phys. 81 (1981), 203-227.

DEPARTMENT OF MATHEMATICS, UNIVERSITY OF MICHIGAN, ANN ARBOR, MICHIGAN 48109

DEPARTMENT OF MATHEMATICS, DUKE UNIVERSITY, DURHAM, NORTH CAROLINA 27706 